\title{
The Small Satellite Integrated Communication Environment (ICE)
}

\author{
John E. Ploschnitznig \\ Riverside Research \\ 15059 Conference Center Dr. Chantilly VA 20151; 703-908-8373 \\ jploschnitznig@riversideresearch.org
}

\begin{abstract}
As the SMALLSAT and NANOSAT communities advance space technology, a communications evolution must also begin. The infrastructure to effectively maintain, monitor, command, and communicate with a satellite requires resources. Historically, this communication link was accomplished by a dedicated ground site with a dish antenna, which provides limited daily access, requires dish operation, and is limited to a single satellite at a time. Although satisfactory for the traditional large satellite architecture, small satellites will break this paradigm as their numbers increase. The value in small satellites may not be in their individual performance, but rather in their numbers. As the number of SMALLSATS and NANOSATS increase from hundreds to thousands of satellites, dedicated ground sites will be rendered ineffective. This paper offers a concept that utilizes existing cell phone technology and a modified cellular infrastructure to revolutionize communications for all satellites. This novel approach will significantly advance satellite technology by providing access to space for academia and entrepreneurs, while offering the military unconventional access to perform its mission.
\end{abstract}

\section{PROBLEM}

With the proliferation of SMALLSAT and NANOSAT satellites, the usual method of communicating with satellite systems, that is, using dedicated ground stations with dish antennas is rendered ineffective. This method provides only limited daily access, requires dish operation, and is limited to a single satellite at a time.

\section{SOLUTION}

The solution is very simple: modify existing cellular towers to provide a fixed upward pointing narrow beam antenna. A single cell tower provides limited communication access, but distributing these antennas over appropriately spaced cell towers provides overlapping coverage spanning large areas of arbitrary size (Figure 1).

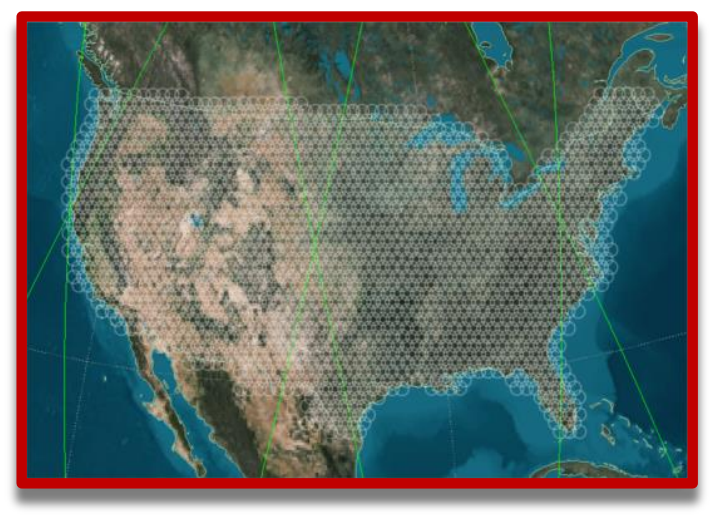

Figure 1 - Illustrates Overlapping Coverage
The goal is to integrate satellite communications into the existing cellular networks and break free from the legacy ground station concept, offering a new Integrated Communication Environment (ICE) for all future satellite programs.

\section{Satellite ICE Module}

Many of today's SMALLSAT and NANOSAT satellite systems use smartphone technology for onboard data processing based on size, sophistication, and needed capabilities. ICE leverages the communication capability of these powerful devices to transmit and receive data to and from the satellite. As the ICE communication technology advances, waiting hours to access the next available ground site will become history and watching live streaming video from space will become the norm.

Smartphone technology is currently being considered for the operating system on many of these new small satellite applications (Figure 2).

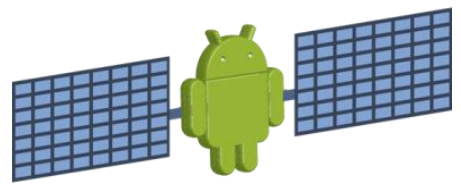

Figure 2 - DROID ${ }^{\mathrm{TM}}$ Integration into Satellite Technology

Although this technology has greatly evolved over the past decade for terrestrial use, some modifications are 
required for a satellite communication implementation. The link budget analysis points to increasing transmit power and adding a downward pointing directional antenna as two variables that can be adjusted in order to secure the link.

\section{ICE Cell Tower}

Current cellular towers are dedicated to providing the necessary coverage for terrestrial communication through antennas aimed at the horizon. ICE requires that a directional upward pointing antenna be integrated on existing cell towers (Figure 3).

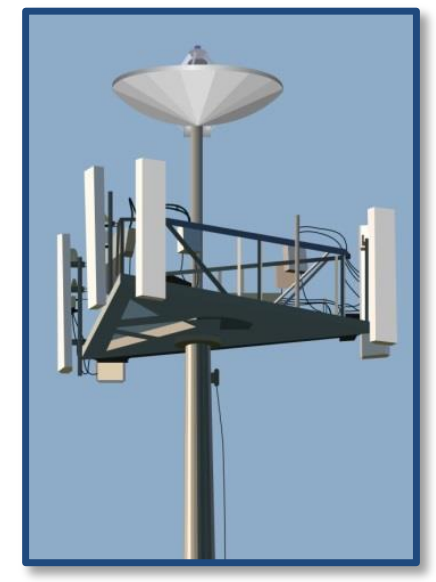

Figure 3: Zenith Antenna Integration

Based on the particular cellular network and available transmit/receive frequencies, an antenna configuration can be designed to provide the required orbital footprint at Low Earth Orbit. For this analysis, we assume a 300 $\mathrm{km}$ altitude orbit. A $4 \mathrm{G}$ network operating at $4 \mathrm{GHz}$ requires a $25 \mathrm{~cm}$ dish (slightly larger to minimize interference with terrestrial antennas) to create an 18 degree footprint in space. This footprint requires an ICE cell tower antenna every $60 \mathrm{~km}$ to provide pattern overlap. The details of the design will vary depending on the selected network and available frequencies.

What makes this approach interesting is a network of many cell towers, appropriately spaced, in order to provide overlapping orbital coverage at Low Earth Orbit (LEO) satellite altitudes. Only a fraction of the many available cell towers are required to provide continual communication over large areas of land.

A thorough link budget analysis is needed to ensure dedicated communications can be established with any satellite passing through the ICE antenna beams. The balance of the analysis defines various factors that can be adjusted to help define the ICE network size, number of antennas, transmit power, receiver sensitivity, and cost to build and install equipment. Ultimately, a solution that provides the largest effective individual footprint will drive the ICE cell tower antenna design since this will result in a lower ICE network cost. A business case must be made which demonstrates the return on investment for populating an ICE cellular network.

\section{The Virtual Ground Site}

The ICE approach relieves the satellite community from depending on traditional dedicated ground sites, and instead a virtual satellite monitoring system will be established. A generic approach will be used to send data to and from the satellite in logically-small transfer packages (ICE Pacs). Based on cell tower access statistics (worst-case short-duration access), packages would be designed to identify standardized data types: Command and Control (C2) uplink; Status of Health (SoH) downlink; and Data Packages (Puzzle Pieces in Figure 4), etc.

Software will be designed to monitor $\mathrm{SoH}$, manage customer needs, perform satellite sensor tasking, create satellite commands and manage satellite operations, operate the ICE Network (under certain operational demands such as Multiple-Cell Tower Operations), and manage the ICE Pacs into logical ICE Trays (Figure 4).

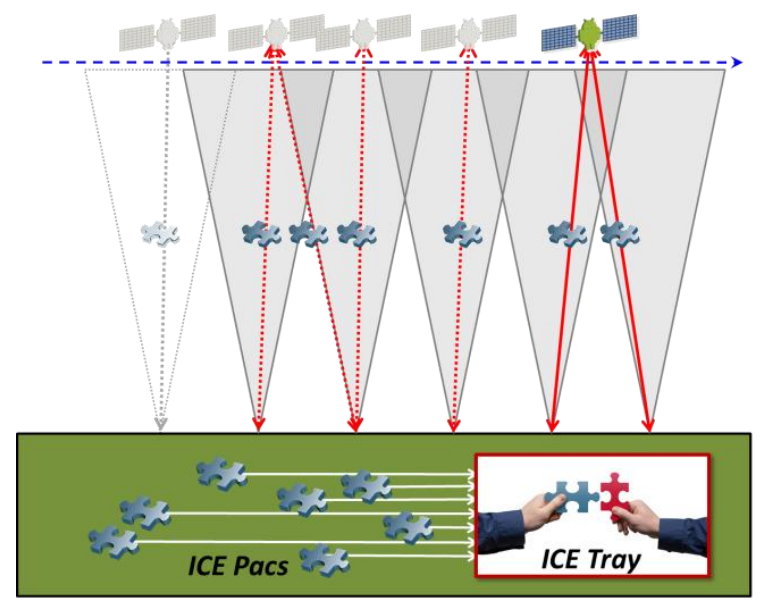

Figure 4: The Virtual Ground Site

Although the existing telecommunication infrastructure will be used to seamlessly integrate the ICE network, there are refinements that can be made to minimize typical cellular communication operational overhead. For example, the fact that we know the satellite trajectory allows us to configure the appropriate sequential cell towers to act as one continuous connection and offer seamless video transmission. 


\section{TECHNICAL ISSUES}

Although this concept leverages the success and maturity of existing cellular network technology, integration of these pieces into the ICE system presents several hurdles: cell phone communication hardware on satellites, satellite antenna and transmitter power requirements, cell tower antenna and associated transmit/receive hardware, selecting the appropriate cellular phone network, software to manage the system, etc. The goal is to make communications achievable and affordable by simply incorporating the necessary cellular equipment into the satellites. Despite the many technical issues that must be resolved, the most difficult challenge is likely to be political: "How do we convince industry to invest in the necessary cellular infrastructure to make the ICE concept feasible?"

\section{Link Budget Analysis}

A link budget analysis is needed to define the ICE configuration parameters (antenna gain, transmit power, receive sensitivity, etc.) of the satellite phone and cell tower system. Currently used cell phone parameters may be modified since they will not be operated in the conventional fashion and will not be restricted by human safety requirements. Likewise, ICE cell tower antennas will be pointing upwards and can incorporate low sidelobe and backlobe antennas. The goal is to ensure feasibility of an executable communication system that provides seamless satellite-to-cell tower communications while operating within FCC approved restrictions.

\section{Antenna Patterns}

The cell tower antenna pattern will set the pace. The antenna will be mounted rigidly on top of the cell tower and be pointing upwards. This will minimize interference with the many other antennas in the vicinity of the cell tower. The antenna beam width is a critical operations and cost driving factor. It defines the orbital intercept area in space and the maximum distance between ICE antenna installations.

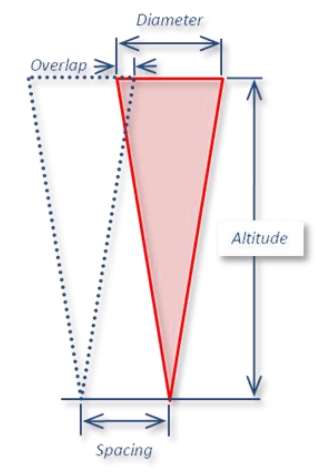

Figure 5: Antenna Design Considerations
These physical settings establish the satellite operating environment (access time per cell), which impact communication operations (ICE Pac size, etc.).

The link budget analysis will determine whether any candidate configuration is feasible. The desire will be to minimize the number of ICE cell tower antennas needed to perform the mission, thus minimizing ICE hardware installation costs (Figure 6).

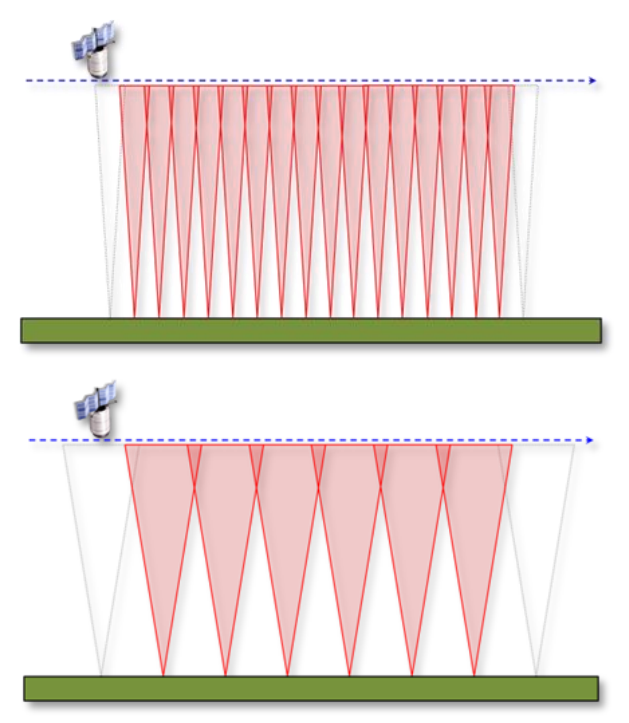

Figure 6: Antenna Gain Performance vs Spacing

Likewise, the satellite ICE communication antenna must be designed to support simultaneous coverage over neighboring cell towers to ensure uninterrupted communications (Figure 7).

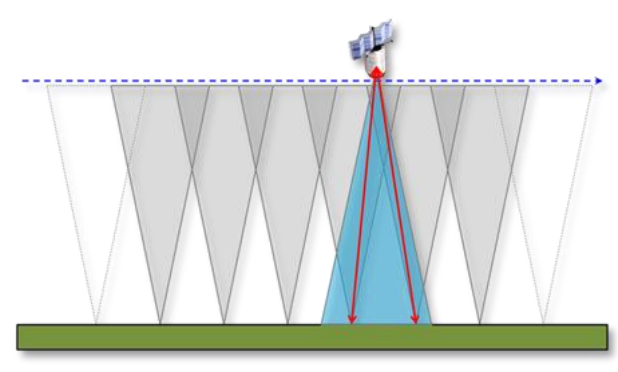

Figure 7: Simultaneous Coverage

In short, the link budget analysis will help define minimum equipment requirements and performance levels to ensure a robust communication capability.

\section{Cellular Networks}

Cell phone services have significantly evolved over the past decade. Advances in telecommunication technology have transformed a simple voice phone into a media storefront. Networks are now able to stream 
video into the palm of your hand. These advances should also benefit the small satellite community.

What service is the best: AT\&T ${ }^{\mathrm{TM}}$, Verizon ${ }^{\mathrm{TM}}$, Sprint ${ }^{\mathrm{TM}}$, 3G, 4G, GSM? Each has benefits and shortcomings, but the key factors that will determine the initial choice will be the service that offers a programmable interface, can be used globally, and offers low operating costs. Since many of the satellites that will use ICE are for research and academia, the cell network of choice will be the one which offers the most flexibility for the satellite owner/operator. Open architectures allow academia and commercial industry to explore various communications concepts for their satellite applications. The cost of the ICE cell tower equipment (antenna, receiver, transmitter, cell tower integration, etc.) must be minimized. Non-recurring costs associated with the initial design and fabrication should be subsidized by the federal government to reduce the upfront investment (similar to government's role in developing the internet and GPS).

The ultimate goal is to allow a satellite vendor to purchase a satellite-enabled phone that can be integrated into their satellite hardware. To reduce weight, these phones would offer no video interface or antenna, and these phone circuit cards could be easily integrated into a NANOSAT or SMALLSAT chassis. A monthly service fee or data-package fee could be charged, and at least initially, a usage fee may apply.

\section{ICE Software}

This is a major R\&D growth area. Firmware is necessary to translate remote commands into satellite operations. Software is necessary to manage satellite tasking, the communication network, and information flow to/from the satellite (Figure 8).

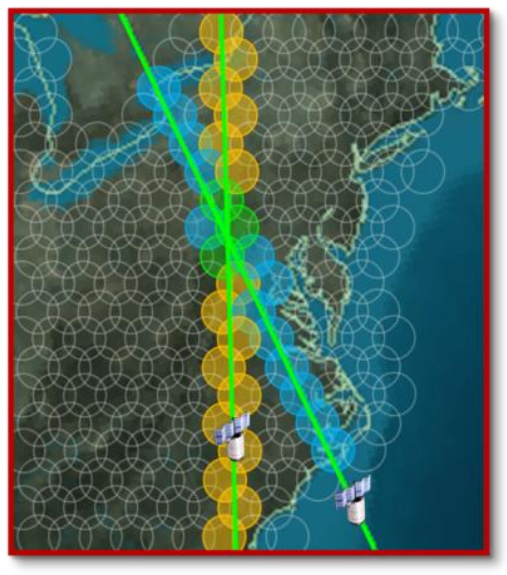

Figure 8: ICE Operations Management
Applications will be developed for handheld devices to request collection tasking and to view products.

Ultimately, the goal is to provide a framework, an architecture (Figure 9), from which academia, commercial industry, or even the ordinary citizen can explore satellite technology. Eliminating the physical ground site and offering a virtual communication framework unleashes the public to explore and invent in an area that was previously controlled by a limited and well-funded private community.

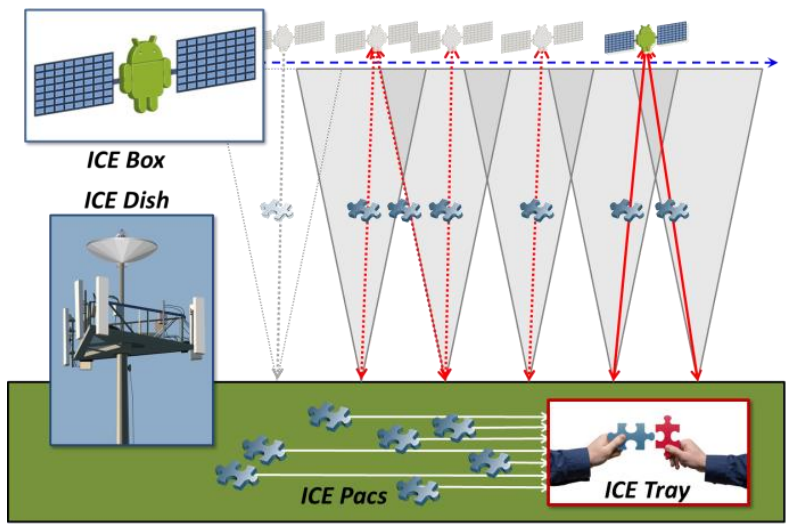

Figure 9: ICE Architecture

\section{APPLICATIONS}

The applications are many and include homeland security, emergency management, natural disaster, monitoring, communication, atmospheric and ionospheric research, sensor calibration, forest fires, weather, land survey, etc. There are many concepts for SMALLSATs, NANOSATs, and PICOSATs, from single satellites to constellations of tens to hundreds of satellites. Legacy communication systems and single pedestal antennas would be overwhelmed by the increased demands associated with managing thousands of satellites and systems, monitoring their health, commanding each satellite, and managing the flow of data/information to and from each satellite. This is the problem that the ICE concept addresses. The lessons learned from today's cellular communication networks in simultaneously managing thousands of phone calls will be used to manage the information to and from these many satellites.

\section{“Look at me"}

Let's assume we have a constellation of 100+ satellites (multiple sun-synchronous orbital planes with many satellites in each plane). Such a constellation would offer on-demand coverage over large areas of land. These satellites could be configured in many ways (video, Electro Optical, Thermal, Hyper/Multi Spectral, etc). With the click of a button on a handheld phone 
application - "Look at me" - the phone geo-location would be sent to the task manager and would designate the appropriate satellite(s) to point towards the uploaded latitude and longitude (Figure 10).

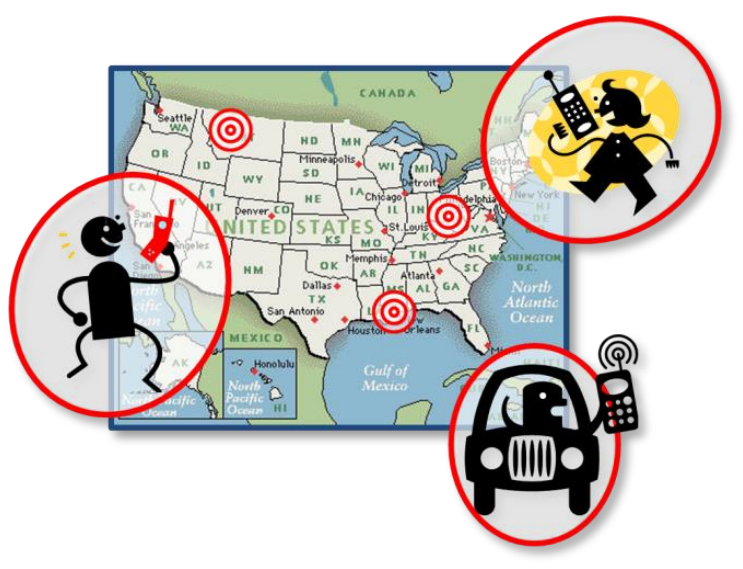

Figure 10: "Look at me" Application

The application for this could be used many ways: natural disaster, news, tornado sightings, etc. Anyone could request snapshots and videos through a simple application or even on the computer by simply clicking the map. Immediately, the ICE Tasking Management System would task available satellites supporting the "Look at me" service.

\section{The Dynamic Earth}

Videos could be painted onto any global web service: Google Earth ${ }^{\mathrm{TM}}$, NASA World Wind, CESIUM, etc. These form the digital canvas upon which to paint information from hundreds and thousands of data sources. Imagine 100 satellites feeding streaming video onto this global canvas. This application would allow users to $\log$ in and task a satellite via a simple pointand-click interface.

\section{Military Application}

The government may choose to encrypt their data (eICE Pac or Black ICE) to protect the content of all uplink and downlink data. In certain cases, the government may utilize ICE as a way to transmit satellite live data to designated receive sites, with this data then forwarded to appropriate person(s) through established secure means. USSOCOM would be a likely customer in such cases.

The US State Department may utilize such a system to monitor international activities in such global hotspots as Libya, Egypt, Syria, Yemen, etc. This would provide real-time situational awareness on large scale international activities. This effectively becomes an eye in the sky (albeit with limited resolution).
There are many different applications that can be attempted at very low cost. Space Situational Awareness, signals monitoring, hyper-spectral, weather, etc. Whatever the technology, ICE offers a robust communication infrastructure to get the data down quickly.

\section{CONCLUSION}

The overall ICE benefit is to provide large area unrestricted satellite communications using relatively simple, inexpensive, redundant, and scalable technology. Utilizing the existing cellular network leverages the infrastructure of a massive data communications network to disseminate the information. As the numbers of small satellites and constellations increases, this approach offers unlimited access. It can be applied globally on cell towers or at any broadband internet access point. The legacy dish pedestal approach can only handle a single satellite within its field of view, whereas ICE can simultaneously handle many satellites with a single cell tower; no pointing, no tracking, no prioritizing, no manin-the-loop. Just as the internet and cell phone technology offered people unrestricted access to each other, ICE extends the reach of these two technologies and offers unrestricted access to space.

Riverside Research has a rich history of bringing technology-based solutions to our customers in the most cost effective manner, especially in the satellite collection tasking and mission management arena. As a not-for-profit corporation, Riverside Research has supported the US Government for over four decades and looks forward to meeting future challenges.

Further details regarding the ICE system and technology are disclosed in U.S. Patent No. 8,751,064 B2 entitled "Methods and Systems for Satellite Integrated Communications," which issued June 10, 2014. 